\title{
Selective Synthesis of Polyoxyethylene-Polyoxypropylene Block Copolymer (Poloxamer) Fatty Acid Monoesters Over Homogeneous Organotin Catalyst
}

\author{
Janusz Nowicki ${ }^{1} \cdot$ Julia Woch $^{1} \cdot$ Małgorzata Mościpan $^{1} \cdot$ Andrzej Robaszkiewicz $^{1}$. \\ Rafał Grabowski $^{1} \cdot$ Izabela Semeniuk $^{1} \cdot$ Karol Erfurt $^{2}$
}

Received: 7 October 2016/ Accepted: 16 September 2017/Published online: 26 September 2017

(c) The Author(s) 2017. This article is an open access publication

\begin{abstract}
The synthesis of selected polyoxyethylenepolyoxypropylene block copolymer (poloxamer) fatty acid monoesters is presented. Organotin homogeneous catalyst Sn bis(2-ethylhexanoate) effectively catalyzed the esterification reaction of (EO)-(PO)-(EO) block copolymer (poloxamer) with fatty acids. The reaction proceeded in high yield and high selectivity to monoesters. Content of diesters in final products was below $1 \mathrm{wt} \%$. The new protocol opened up a high yield and high selective method for the synthesis of poloxamer fatty acid monoesters. These products are potentially interesting for industrial applications, e.g. in lubricants, cosmetics and, in particular, as potential emulsifying agents compatible with hydrocarbon bases, such as paraffin.
\end{abstract}

Keywords Poloxamer - Esterification · Monoesters · Fatty acid - Organotin catalyst

\section{Introduction}

Polyoxyalkylenene glycol esters are a group of nonionic surfactants with a vast field of application. Polyoxyethylene and polyoxypropylene glycol fatty acid esters,

Electronic supplementary material The online version of this article (doi:10.1007/s11743-017-2020-2) contains supplementary material, which is available to authorized users.

Janusz Nowicki

nowicki.j@icso.com.pl

1 Institute of Heavy Organic Synthesis "Blachownia", Kędzierzyn-Koźle, Poland

2 Faculty of Chemistry, Silesian University of Technology, Gliwice, Poland prepared by esterification of fatty acids with corresponding polyoxyalkylene glycols, are classified as non-toxic and non-irritating nonionic emulsifiers. Depending on their molecular weight and water solubility, esters can be applied in both aqueous and non-aqueous systems. Polyoxyalkylene esters of fatty acids are widely used as emulsifiers in cosmetics and textiles [1, 2]. Fatty acid polyol esters have also been recently used for removal of soil (or water) contamination caused by spilled oil. Herein, their relatively low toxicity for marine fauna plays a significant role [3, 4]. Important representatives of discussed group-laurates, stearates and oleates, primarily used as emulsifiers in cosmetics and pharmaceutics, are also applied as components for drilling and cutting oils [5]. Application as lubricants and filament cohesion agents for fiber finishing is less relevant, because of their limited solubility in water.

Polyol esters are produced by chemically catalyzed esterification [6, 7]. Fatty acid esters of polyoxyethylene glycols, in general, are prepared either via oxyethylation or esterification route [8]. For increased selectivity of monoesters, a large excess of polyoxyethylene glycol to fatty acid, even 6-12 molar ratio, is recommended [9]. After the reaction, excess of polyoxyethylene glycol is washed out with concentrated salt solution [10]. The original method of the selective synthesis of monoesters using boric acid was reported by Hartman, but this method is of little preparative significance [11]. Many of the esterification reactions are carried out at elevated temperatures in presence of homogeneous acidic, amphoteric or basic catalysts [12]. Sodium or potassium hydroxide or $p$ toluenesulphonic acid are the most preferred catalysts. In order to favorably shift the equilibrium, esterification water is removed by applying vacuum or using a flow (stream) of nitrogen. 
Fatty acid esters of polyoxyethylene glycols can be also produced from fats or plant oils in transesterification processes in presence of strong basic catalysts. The reaction product in general is a mixture of mono-, di- and triglycerides, glycerol, unreacted polyoxyethylene glycol and also a mixture of mono- and diesters [13, 14]. Because of the very complex character of the product this method is not preferred.

Diesters of polyalkylene glycols can be selectively synthesized with high yields by using a high molar excess of fatty acid. Synthesis of monoesters, which are characterized by more interesting surface active properties (e.g. as emulsifier for textile industry) [15], is possible, but requires the use of high molar excess of glycol [16]. Equal activity of both hydroxyl groups in polyoxyethylene glycols leads to esterification of both of hydroxyl groups. As reported by Corma et al. better miscibility of fatty acids with the polyoxyalkylene glycol monoesters compared to glycol promotes the reaction of fatty acids with hydroxyl group of monoesters, rather than with polyoxyethylene glycol, which is immiscible with fatty acids. In addition to that, disproportionation of monoester formed in the first step by transesterification also leads to increased amounts of diester [17].

Fatty acid monoesters of poly(oxyalkylene) glycols can be also obtained in oxyalkylation route. Direct oxyalkylation of fatty acids with conventional basic catalysts yields a complex mixture of mono- and diesters, as well as various polyethylene glycols as by-products, with a wide range of polyethylene glycol units. The final product can be used as emulsifiers in food, cosmetics and other technical applications $[18,19]$.

In recent years, enzyme-catalyzed high yields synthesis of fatty acid esters and fatty acids and polyoxyalkylene glycols through esterification and interesterification processes have attracted particular attention. The catalytic activity of lipases toward hydroxy fatty acid esters was well studied by Hayes [20-23]. In the esterification of several hydroxyl fatty acids Rhizomисо miehei lipase was also adopted [24-27]. Steffen et al. used $R$. miehei and Candida antarctica lipases as biocatalyst for the synthesis of monoglycerides of 17-hydroxy- and 12-hydroxystearic acids with high yield [27]. A promising alternative for this processes could be the use of homogeneous organometallic Lewis compounds as catalysts, which are used in many other commercial processes, e.g. synthesis of polyesters. Our previous experiments on the esterification reaction of higher fatty acids and polyols conducted over various Ti, $\mathrm{Zr}$ and $\mathrm{Sn}$ catalysts have shown that homogeneous $\mathrm{Sn}$ bis(2-ethylhexanoate) is characterized by the highest activity and selectivity to the desired products [28].

In this paper, the novel high selective method of the synthesis of monoesters of selected hydrophobic polyoxyalkylene glycols-polyoxyethylene-polyoxypropylene block copolymers (poloxamer) is presented. In the literature, there is practically no detailed information on the synthesis of poloxamer fatty acid esters. Only in the FDA report can find information, that poloxamer fatty acid esters are safe and can be used to manufacturing materials, that come in contact with food [29]. A homogeneous Sn bis(2ethylhexanoate) catalyst was used. The described method under optimal reaction conditions results in the desired monoesters with high selectivity, above $99 \%$.

\section{Experimental}

Oleic acid $90+\%$ was purchased from Croda Int. as Priolene 6936. Stearic acid $99+\%$, lauric acid $99+\%$ and octanoic acid $99+\%$ were purchased from Sigma Aldrich. Polyoxyethylene-polyoxypropylene-polyoxyethylene block copolymer (poloxamer) was purchased from BASF as Pluronic PE3100 (mol mass $=1000$; molar mass of propylene glycol block $=850$; polyethylene glycol content $=10 \mathrm{wt} \%$; hydroxyl value $=105 \mathrm{mg} \mathrm{KOH} / \mathrm{g}$ ). Commercially available homogeneous $\mathrm{Sn}$ bis(2-ethylhexanoate) purchased from PMC Organometallic as Fascat 2003 was used as catalyst. Cyclohexane and potassium phosphate analytical grade (Pure) were purchased from Sigma Aldrich.

Gel permeation chromatography (GPC) was carried out on a L-7100 series pump (Merck-Hitachi) equipped with degasser (Knauer) and three column series from Polymer Laboratories, Inc. (Amherst, MA, USA) consisting of PLgel $3 \mu \mathrm{m}$ Guard $(7.5 \times 50 \mathrm{~mm})$, PLgel $5 \mu \mathrm{m}$ MiniMIX-E $(7.5 \times 250 \mathrm{~mm}$, molecular weight range $500-30,000 \mathrm{~g} / \mathrm{mol})$ and PLgel $5 \mu \mathrm{m}$ MiniMIX-E $(7.5 \times 250 \mathrm{~mm}$, molecular weight range $500-30,000 \mathrm{~g} / \mathrm{mol}$ ) columns. The system was fitted with a VISCOTEK VE 3580 differential refractometer detector and anhydrous tetrahydrofuran was used as the mobile phase $(0.3 \mathrm{~mL}$ min flow rate). Data were collected and processed using GRAMS/386 for Chromatography software and calibrated against polystyrene standards. Analyses were performed at $30{ }^{\circ} \mathrm{C}$.

${ }^{1} \mathrm{H}-\mathrm{NMR}$ spectra were recorded on Varian Unity Inova Plus spectrometer $\left(\mathrm{CDCl}_{4}, 400 \mathrm{MHz}\right)$ FT-IR spectra were recorded on MATTSON 3000 spectrometer (Unicam) at $500-4000 \mathrm{~cm}^{-1}$. Kinematic viscosity was determined on Brookfield DV-II+ viscometer. Density of esters at $25{ }^{\circ} \mathrm{C}$ was determined using $1 \mathrm{~cm}^{3}$ micro-pycnometer. Hydrophile-lipophile balance values (HLB) of polyol esters were calculated according to Davies method [30].

\section{Synthesis of Polyol Monoesters (General Procedure)}

In a $200 \mathrm{~mL}$ glass reactor, equipped with mechanical stirrer, an electronic temperature controller, glass capillary 
and a receiver to collect esterification water were placed $180 \mathrm{~g}$ of Pluronic PE3100 and the required amount of fatty acid (according to desired COOH:OH molar ratio calculated on the basis of hydroxyl value). The reactor was heated up to $150{ }^{\circ} \mathrm{C}$ and then a proper amount of catalyst was added into the reactor. Temperature was raised to $220{ }^{\circ} \mathrm{C}$ and the reaction mixture was then stirred for $9 \mathrm{~h}$. Nitrogen introduced into the reactor (glass capillary) helps to remove the water and also provides a protective atmosphere. Esterification was considered complete when the acid number of the reaction mixture was below $1 \mathrm{mg} \mathrm{KOH} /$ g. Crude esters were then diluted with cyclohexane in order to decrease the viscosity and density. This solution was washed at first by $5 \mathrm{wt} \%$ of $\mathrm{K}_{3} \mathrm{PO}_{4}$ water solution and twice with deionized water to reach a neutral $\mathrm{pH}$. Solvent was removed under reduced pressure on a rotary evaporator.

\section{Results and Discussion}

It is well known that the esterification reaction is strongly influenced by the efficiency of water removal. In the case of low molecular weight alcohols (methanol, ethanol), which do not form heteroazeotropic mixtures with water, the process is difficult. In such type of alcohols the satisfactory results are achieved by using a large excess of alcohol (e.g. direct esterification of fatty acid with an excess of methanol). A more convenient situation is observed in the case of higher aliphatic alcohols $\left(>\mathrm{C}_{4}\right)$. They form heteroazeotropic mixtures with water, which facilitates the removal of the esterification water. Unfortunately, for higher aliphatic alcohols $\left(>\mathrm{C}_{10}\right)$ and low volatility polyols the above method is not practical. In these cases water can be removed with the use of neutral solvents (e.g. toluene) or by running the esterification reaction at elevated temperatures. This method is applied in the esterification of polyols, also polyoxyalkylene glycols, with fatty acids. However, because of relatively high melting points of polyols and their limited miscibility with fatty acids, an esterification temperature above $150{ }^{\circ} \mathrm{C}$ is required.
To obtain an efficient emulsifying agent, a higher content of monoester in the reaction product is preferred (higher hydrophilicity and higher HLB value). The choice of catalyst and reaction conditions are crucial for high selectivity of monoesters. Monoesters of polyxyalkylene glycols are characterized by better emulsification potential, especially in terms of w/o emulsions. EO/PO/EO block copolymers are a mixture of oligomers with various molecular masses characterized by different values of hydroxyl number.

The aim of this study was too selectively obtain fatty acid monoesters of EO-PO-EO block copolymer (Pluronic PE3100). To find the optimal COOH:OH molar ratio, a series of esterification reactions with different molar ratios were performed. As a control parameter, acid value of the crude ester was adopted. The catalyst (tin octanoate) did not affect the overall acid number in post-synthesis mixtures, so the acid value of the esterification product catalyzed by metalorganic Lewis catalysts depended mainly on the free fatty acid content.

Experiments were conducted using fatty acid: $\mathrm{OH}$ ratio of 1:0.45-1:0.55 in the presence of Sn bis(2-ethylhexanoate) as a catalyst at $220{ }^{\circ} \mathrm{C}$. The esterification reaction in the above adopted reaction conditions resulted mainly in monoesters according to the scheme presented in Fig. 1. Similarly to esterification of polyols, the molar ratio of fatty acid and $\mathrm{OH}$ group is the key factor affecting the composition of post-synthesis mixtures. In EO-PO-EO block copolymers, the terminal groups are primary hydroxyl groups, so this type of polyols in esterification reaction react similarly to most aliphatic $\alpha, \omega$-glycols. However, polyoxyalkylene glycols are a mixture of glycols with various oxyalkylene units and, in consequence, of various molar mass (Gauss rule).

The amount of added catalyst is considered as one of the important parameter in the esterification reaction. The results of preliminary studies of the esterification reaction of oleic acid and Pluronic PE3100 in relation to the amount of added Sn catalyst are presented in Table 1 . The amount of added catalyst had noticeable less importance on final acid values of crude reaction product. Acid value of crude esters obtained for the amount of catalyst at the level

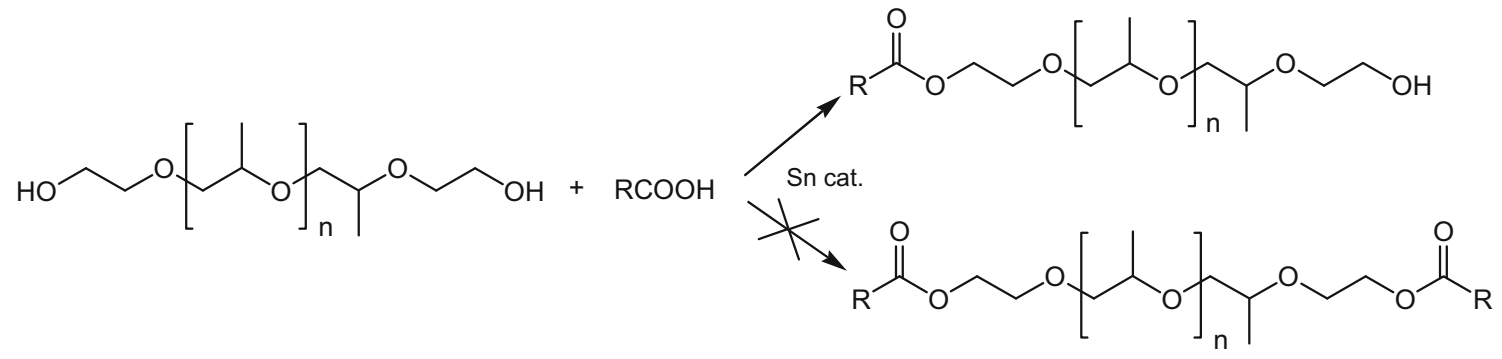

Fig. 1 Synthesis of fatty acid EO-PO-EO block copolymer glycol monoesters 
Table 1 The results of the esterification reaction of oleic acid and Pluronic PE3100 (catalyst amount effect)

\begin{tabular}{ll}
\hline catalyst amount, wt\% & Acid value, $\mathrm{mg} \mathrm{KOH} / \mathrm{g}$ \\
\hline 0.5 & 0.98 \\
0.7 & 0.54 \\
0.9 & 0.50 \\
\hline
\end{tabular}

Reaction conditions: temp. $-220{ }^{\circ} \mathrm{C}$; reaction time $-9 \mathrm{~h} ; \mathrm{COOH}: \mathrm{OH}$ molar ratio-0:0.45

Table 2 The results of the esterification reaction of oleic acid and Pluronic PE3100 (reactants molar ratio effect)

\begin{tabular}{lll}
\hline COOH:OH molar ratio & Monoester, wt $\%$ & Diester, wt $\%$ \\
\hline $0: 0.55$ & 95.5 & 4.5 \\
$0: 0.5$ & 97.4 & 2.6 \\
$0: 0.45$ & 99.0 & 1.0 \\
\hline
\end{tabular}

Reaction conditions: temp. $-220{ }^{\circ} \mathrm{C}$; time $-9 \mathrm{~h}$; catalyst amount$0.7 \mathrm{wt} \%$

$0.7-0.9 \mathrm{wt} \%$ was very similar, so in the following experiments the catalyst was added at $0.7 \mathrm{wt} \%$ only.

In Table 2 are presented the results of esterification reaction of oleic acid and Pluronic PE3100 in relation to COOH:OH molar ratio. Results presented in Table 2 shows that product containing the higher amount of monoester was obtained for a molar ratio below the theoretical value $(0: 0.5)$. For the molar ratio $\mathrm{COOH}: \mathrm{OH}=0: 0.45$ the product contained almost entirely monoester. Assuming the previously established optimum reaction parameters the esterification of Pluronic PE3100 with the series of fatty acids was then conducted. The results of these experiments are presented in Table 3.

Results presented in Table 3 shows that both the acid values and the mono/diester composition for fatty acids used are similar to oleic acid monoester. The acid value of crude products was $0.5-0.6 \mathrm{mg} \mathrm{KOH} / \mathrm{g}$ and the content of diesters was below $1 \mathrm{wt} \%$. Results presented in Table 3 clearly shows that the use of organotin catalyst in the synthesis of EO-PO-EO block copolymers allows for obtaining monoesters with high selectivity, above $99 \%$. These results, especially the selectivity towards monoesters in the presented case, were significantly higher than results described in the literature [15]. In the cited study polyoxyethylene glycol was used as polyol. EO-PO-EO glycols contain terminal hydroxyethyl groups, so they can be considered as polyoxythylene glycols analogs.

The main analytical method widely adopted for determination of oxyalkylene fatty acid ester is gel permeation chromatography (GPC), also used in this study [31]. The results of the esterification of Pluronic PE3100 with oleic acid is presented in Fig. 2 (for GPC analysis of all esters see Supporting Information). As demonstrated in Fig. 2, the crude post-reaction mixture contains only one type of compounds (mixture of glycol monoesters). In order to confirm the contents of reaction products, the reaction mixture was compared with the raw materials used in the synthesis (polyol, fatty acids). Figure 2 clearly shows that the product of esterification consists mainly of monoesters. Small amount of diesters were detected in the case of oleic acid esterification. The lack of free oleic acid confirms high conversion rate of the fatty acid.

Crude esters were purified in order to remove the catalyst and to decrease the amount of free fatty acids in the product. Among various washing methods, the one incorporating a dilute solution of $\mathrm{K}_{3} \mathrm{PO}_{4}$ was adopted. The amount of basic phosphate was a three-fold molar excess relative to the acid values of the crude esters. This method was considered as the best for purification of crude fatty acid esters of various polyols [28].

Chemical structures of obtained esters were confirmed by instrumental analysis. The ${ }^{1} \mathrm{H}-\mathrm{NMR}$ spectra recorded for EO-PO-EO glycol were typical for analogous fatty acid esters. In ${ }^{1} \mathrm{H}-\mathrm{NMR}$ spectra recorded for Pluronic PE3100 fatty acid monoesters several characteristic signals (ppm): 0.88 (terminal - $\mathbf{C H}_{3}$ ); 2.30 (ester $-\mathrm{CH}_{\mathbf{2}} \mathrm{CO}$-); 4.22 (ester - $\left.\mathrm{CO}-\mathrm{OCH}_{2}-\right)$ and additional $5.34(-\mathrm{CH}-\mathrm{CH}-$ ) for oleic acid ester can be found (see Supporting Information). ${ }^{1} \mathrm{H}-\mathrm{NMR}$ spectrum of polyoxyalkylene glycol oleic acid ester is very similar to another oleic acid esters [31].

In the FT-IR spectra recorded for Pluronic PE3100 fatty acid monoesters, several characteristic bonds can be found. The strong intensity $\mathrm{OH}$ stretching vibrations in the region
Table 3 Analysis of crude fatty acid Pluronic PE3100 monoesters

\begin{tabular}{llcr}
\hline Fatty acid & Acid value, $\mathrm{mg} \mathrm{KOH} / \mathrm{g}$ & Monoester, wt\% & Diester, wt\% \\
\hline Octanoic acid & 0.55 & 99.2 & 0.8 \\
Lauric acid & 0.50 & $\sim 100$ & $<0.5$ \\
Oleic acid & 0.54 & 99.0 & 1.0 \\
Stearic acid & 0.60 & $\sim 100$ & $<0.5$ \\
\hline
\end{tabular}

Reaction conditions: temp. $-220^{\circ} \mathrm{C}$; reaction time $-9 \mathrm{~h}$; $\mathrm{COOH}: \mathrm{OH}$ molar ratio-0:0.45; catalyst amount $-0.7 \mathrm{wt} \%$ 


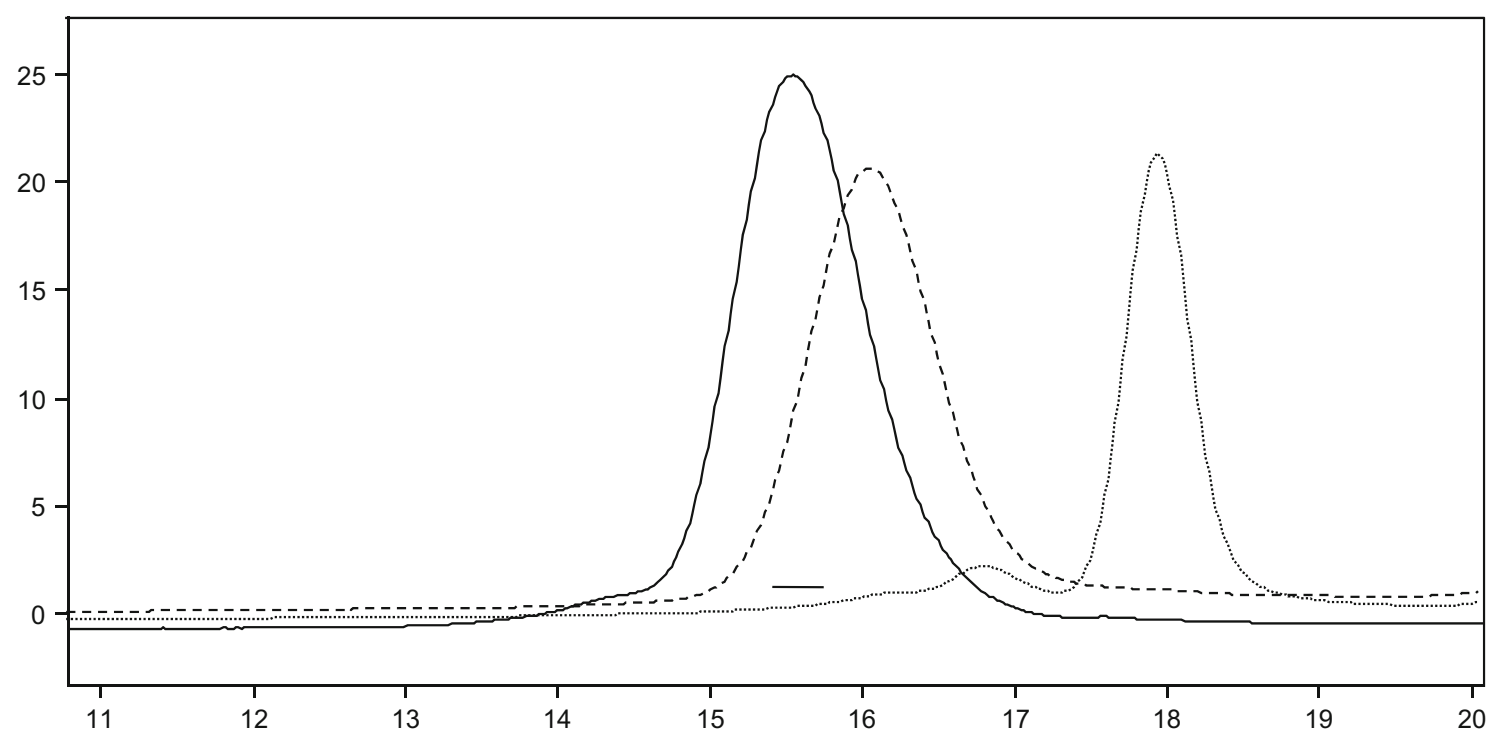

Fig. 2 GPC chromatograms of Pluronic PE 3100 monooleate (line), Pluronic PE 3100 (dashed line) and oleic acid (dotted line)

Table 4 Physicochemical characteristic of purified fatty acid Pluronic PE3100 monoesters

\begin{tabular}{lllll}
\hline Fatty acid & Acid value, $\mathrm{mg} \mathrm{KOH} / \mathrm{g}$ & Density, $\mathrm{g} / \mathrm{cm}^{3}$ & Viscosity, $\mathrm{mPa} / \mathrm{s}$ & HLB \\
\hline Octanoic acid & 0.25 & 0.878 & 100.0 & 7.36 \\
Lauric acid & 0.20 & 0.882 & 137.5 & 5.55 \\
Oleic acid & 0.24 & 0.879 & 152.5 & 2.70 \\
Stearic acid & 0.20 & 0.880 & 148.7 & 2.70 \\
\hline
\end{tabular}

of $3485-3475 \mathrm{~cm}^{-1}$ corresponding to terminal $\mathrm{OH}$ groups confirm the presence of glycol monoesters. The strong intensity bond at $1736 \mathrm{~cm}^{-1}$ corresponds to ester carbonyl group. The strong intensity bond at the region $1112-1109 \mathrm{~cm}^{-1}$ corresponds to polyol ether groups. In all FTIR spectra two characteristic bonds at 1015 and $929 \mathrm{~cm}^{-1}$ are observed that corresponds to oxypropylene segment (see Supporting Information).

Density of purified esters was at the level of $0.878-0.882 \mathrm{~g} / \mathrm{cm} 3$. Kinematic viscosity of Pluronic PE3100 monoesters depended on the fatty acid used. Viscosity values, measured at $25{ }^{\circ} \mathrm{C}$, ranged from 100 to $152 \mathrm{mPa}$ s. Physicochemical data of Pluronic PE3100 fatty acid monoesters are presented in Table 4.

HLB values of synthesized monoesters were determined according to the modified additive Davies method. For surfactant containing $\mathrm{CH}_{2}$, EO, $\mathrm{PO}$ can be written as:

$$
\begin{aligned}
\mathrm{HLB}= & 7+N\left(\mathrm{CH}_{2}\right) \times n \mathrm{CH}_{2}+N(\mathrm{EO}) \times n(\mathrm{EO})+N(\mathrm{PO}) \\
& \times n(\mathrm{PO})+\Sigma \text { other hydrophilic group } \\
& +\Sigma \text { other hydrophobic groups, }
\end{aligned}
$$

where $N\left(\mathrm{CH}_{2}\right), N(\mathrm{EO})$, and $N(\mathrm{PO})$ are the number of groups $\mathrm{CH}_{2}$, EO and PO. $n\left(\mathrm{CH}_{2}\right), n(\mathrm{EO})$ and $n(\mathrm{PO})$ represent the chain lengths for $\mathrm{CH}_{2}, \mathrm{EO}$ and $\mathrm{PO}$ groups. Beside of $\mathrm{CH}_{2}$, EO and $\mathrm{PO}$ groups, Pluronic monoesters contains also ester $\mathrm{C}(\mathrm{O}) \mathrm{O}$ and $\mathrm{OH}$ group as other hydrophilic group and terminal $\mathrm{CH}_{3}$ as other hydrophobic groups. Above equation for synthesized esters can be written as:

$$
\begin{aligned}
\mathrm{HLB}= & 7+N\left(\mathrm{CH}_{2}\right) \times n\left(\mathrm{CH}_{2}\right)+N(\mathrm{EO}) \times n(\mathrm{EO}) \\
& +N(\mathrm{PO}) \times n(\mathrm{PO})+N(\mathrm{COO})+N(\mathrm{OH}) \\
& +N\left(\mathrm{CH}_{3}\right) .
\end{aligned}
$$

$N$ values have been adopted from widely published data as follow: $N(\mathrm{EO})=0.33 ; N($ ester $)=2.4 ; N(\mathrm{OH})=1.9$; $N(\mathrm{PO})=-0.15 ; N\left(\mathrm{CH}_{x}\right)=-0.475[30,32]$. The results of HLB calculations were collected in Table 4. According to widely adopted applications for nonionic surfactants within the HLB ranges, octanoic and lauric acid monoester of Pluronic PE3100 can be classified as W/O emulsifier and oleic and stearic acid monoester as defoamers [33].

\section{Conclusions}

The results described in this study show that homogeneous organotin catalyst is characterized by high activity and selectivity in the synthesis of fatty acid monoesters of Pluronic PE3100 EO-PO-EO block copolymer. Sn bis(2ethylhexanoate) proved to be useful in the synthesis of 
oxyalkylene glycols with longer fatty acid chains that are potentially suitable as W/O emulsifier and defoamers. In optimal reaction conditions polyoxyalkylene glycol fatty acid esters with high selectivity to monoesters (99\%) were obtained. Simple synthesis procedure, relatively mild reaction conditions, commercial availability of catalyst and also no need for difficult and expensive purification stages enable, in our opinion, application of this method in industrial scale.

Acknowledgements The authors wish to thank the National Centre for Research and Development (Poland) for providing the financial support for the realization of these studies. This work has been done within the BIOSTRATEG II (ID 298537/16) project "New types of food packages comprising renewable raw materials and innovative paraffin-based impregnates".

Open Access This article is distributed under the terms of the Creative Commons Attribution 4.0 International License (http://crea tivecommons.org/licenses/by/4.0/), which permits unrestricted use, distribution, and reproduction in any medium, provided you give appropriate credit to the original author(s) and the source, provide a link to the Creative Commons license, and indicate if changes were made.

\section{References}

1. Winter R. A consumer's dictionary of cosmetic ingredients: complete information about the harmful and desirable ingredients found in cosmetics and cosmeceuticals. New York: Three Rivers Press; 2005.

2. Panda H. The complete book on textile processing and silk reeling technology. Delhi: Asia Pacific Business Press Inc.; 2010.

3. ICI, GB patent 1,338,385, dispersion of oil slicks (1970/1973).

4. Maag H. Fatty acid derivatives: important surfactants for household, domestics and industrial purposes. J Am Oil Chem Soc. 1984;61:259-67.

5. Meffert A. Technical uses of fatty acid esters. J Am Oil Chem Soc. 1984;61:255-8.

6. Shaw JF, Lo S. Production of propylene glycol fatty acid monoesters by lipase-catalyzed reactions in organic solvents. J Am Oil Chem Soc. 1994;71:715-9.

7. Mattson FH, Volpenhaim RA. Synthesis and properties of glycerides. J Lipid Res. 1962;3:281-96.

8. Kosswig K. Polyoxyethylene esters of fatty acids. In: van Os NM, editor. Nonionic surfactants: organic chemistry. Surfactant science series, vol. 72. New York: Marcel Dekker; 1998. p. 123-46.

9. Weil JK, Koos RE, Linfield WM, Parris N. Nonionic wetting agents. J Am Oil Chem Soc. 1979;56:873-7.

10. Schutte H, Schoeller C, Wittwer M. Production of new high molecular sulfur-containing condensation products. US patent 2,129,709 I.G. Farbenindustrie A.G (1938).

11. Hartman L. Glyceride synthesis by direct esterification. J Chem Soc (London). 1957;1957:3572-5.

12. Goldsmith HA. Polyhydric alcohol esters of fatty acids. Their preparation, properties, and uses. Chem Rev. 1943;33:257-349.

13. Schuchardt U, Sercheli R, Vargas RM. Transesterification of vegetable oils: a review. J Braz Chem Soc. 1998;9:199.

14. Jiratumnukul N, Van De Mark MR. Preparation of glycol esters of soybean oil fatty acids and their potential as coalescent aids in paint formulations. J Am Oil Chem Soc. 2000;77:691-7.
15. Weipert EA. US patent 4,169,062, random copolymers of polyoxyethylene polyoxypropylene glycol monoester, process of making the same and textile fiber containing the same (1979).

16. Hamid SBA, Abdullah FZ, Ariyanchira S, Mifsud M, Iborra S, Corma A. Polyoxyethylene esters of fatty acids: an alternative synthetic route for high selectivity of monoesters. Catal Today. 2004;97:271-6.

17. Parris N, Weil JK. Effect of polyoxyethylated materials on the interaction of surfactants with skin. J Am Oil Chem Soc. 1979;56:776-82.

18. O'Lenick AJ Jr, Parkinson JK. The effect of branching and unsaturation upon some properties of polyoxyethylene glycol diesters. J Surfactants Deterg. 1998;1:529-32.

19. O'Lenick AJ Jr. Evaluation of polyoxyethylene glycol esters of castor, high-erucic acid rapeseed, and soybean oils. J Surfactants Deterg. 2000;3:201-6.

20. Hayes DG, Kleiman R. Lipase catalyzed synthesis and properties of estolides and their esters. $\mathrm{J}$ Am Oil Chem Soc. 1995;72:1309-16.

21. Hayes DG. The catalytic activity of lipases toward hydroxy fatty acids-a review. J Am Oil Chem Soc. 1996;73:543-59.

22. Hayes DG, Kleiman R. Lipase-catalyzed synthesis of lesquerolic acid wax and diol esters and their properties. J Am Oil Chem Soc. 1996;73:1385-92.

23. Hayes DG, Gulari E. Formation of polyol fatty acid by lipases in reversed micelles. Biotechnol Bioeng. 1992;10:110-8.

24. Ghosh Ray S, Bhattacharyya DK. Enzymatic preparation of ricinoleic acid esters of long-chain monoglyceride alcohols and properties of the esters. J Am Oil Chem Soc. 1992;69:85-8.

25. Chattopadhyay S, Mamdapur VR. Enzymatic esterification of 3-hydroxy butyric acid. Biotechnol Lett. 1992;15:245-50.

26. Wagner F, Kleppe F, Lokotsch W, Ziemann A, Lang S. Synthesis of uncommon wax esters with immobilized lipases. Ann N Y Acad Sci. 1992;672:484-90.

27. Lang S, Multzsch R, Passeri A, Schmeiche A, Steffen B, Wagner F, Hamman D, Cammenga HK. Unusual wax esters and glycolipids: biocatalytic formation and physicochemical characterization. Acta Biotechnol. 1991;11:379-86.

28. Nowicki J, Stańczyk D, Drabik J, Mosio-Mosiewski J, Woszczyński P, Warzała M. Synthesis of fatty acid esters of selected higher polyols over homogeneous metallic catalysts. J Am Oil Chem Soc. 2016;93:973-81.

29. GRAS Notice 000433: poloxamer fatty acid esters, Food and Drug Administration Report, April 20, 2012, USA.

30. Guo X, Rong Z, Ying X. Calculation of hydrophile-lipophile balance for polyethoxylated surfactants by group contribution method. J Colloid Interface Sci. 2006;298:441-50.

31. Schmit TE. Characterization of nonionic surfactants. In: Schmitt TE, editor. Analysis of surfactants. New York: Marcel Dekker; 2001.

32. Sowada R, McGowan JC. Calculation of HLB values. Tenside Surfactants Deterg. 1992;29:109-13.

33. Edens MW. Application of polyoxyalkylene block copolymers. In: Nace V, editor. Nonionic surfactants: polyoxyalkylene block copolymers. Surfactant Science Series, vol. 60. New York: CRC Press; 1996. p. 185-210.

Janusz Nowicki assistant professor in the Institute of Heavy Organic Synthesis "Blachownia", Kędzierzyn-Koźle, graduated from Wrocław University of Technology, Wrocław, Poland, in 1978. He completed his Ph.D. in chemistry from Łódź University of Technology, Łódź, and D.Sc. degree in chemical technology from Wrocław University of Technology, Wrocław, Poland. Currently, he works as a senior researcher in the Renewable Raw Materials Department. He has co-authored 70 original papers. The main areas of his scientific 
interest are chemistry of renewable raw materials, modern heterogeneous catalysis, and synthesis and application of ionic liquid. He is a member of the Polish Chemical Society.

Julia Woch graduated from Silesian University of Technology, Faculty of Chemistry, in 2010. She is a researcher in the Institute of Heavy Organic Synthesis "Blachownia" in Kędzierzyn-Koźle, Poland, and a Ph.D. student in the Opole University. The field of her studies covers aggregation of surfactants in aqueous solutions and chemistry of industrial colloids.

Małgorzata Mościpan graduated from the Opole University in 2009 (Faculty of Chemistry). She received her Ph.D. degree in analytical chemistry in 2013. She works in the Analytical Department of the Institute of Heavy Organic Synthesis "Blachownia". Her research work is concentrated on developing analytical methods for the determination of the analyte in various types of samples.

Andrzej Robaszkiewicz graduated from the University of Science and Technology in Bydgoszcz, Poland, in 1998. He completed his
Ph.D. in chemistry from the Gdańsk University of Technology, Gdańsk, Poland, in 2004. He works in the Institute of Heavy Organic Synthesis "Blachownia" in Kędzierzyn-Koźle, Poland, as a research assistant. His work focuses on organic synthesis and chemical technology.

Rafał Grabowski graduated from the Silesian University of Technology, Faculty of Chemistry, in 2013. He works in the Institute of Heavy Organic Synthesis "Blachownia" in Kędzierzyn-Koźle, Poland, as a research technical specialist. His work focuses on organic synthesis and chemical technology.

Izabela Semeniuk graduated from the Silesian University, Departament of Biochemistry, Katowice, in 1987. She specializes in spectroscopic techniques.

Karol Erfurt graduated from the Silesian University of Technology, Faculty of Chemistry, in 2011. After graduation, he remained at the SUT as a technical worker. In his work, he deals with the synthesis of ionic liquids based on natural compounds. 